Ciência Florestal, Santa Maria, v. 21, n. 2, p. 183-192, abr.-jun., 2011

ISSN 0103-9954

\title{
REGENERAÇÃO NATURAL AVANÇADA DE ESPÉCIES ARBÓREAS NATIVAS NO SUB-BOSQUE DE POVOAMENTOS DE Eucalyptus saligna Smith., NA ZONA DA MATA SUL DE PERNAMBUCO
}

\author{
ADVANCED NATURAL REGENERATION OF NATIVE ARBOREAL SPECIES IN THE \\ SUB-FOREST OF SETTLEMENTS OF Eucalyptus saligna Smith., IN THE AREA OF THE SOUTH \\ FOREST OF PERNAMBUCO
}

\author{
Antonia Lidiane de Alencar ${ }^{1}$ Luiz Carlos Marangon ${ }^{2}$ Ana Lícia Patriota Feliciano ${ }^{3}$ \\ Rinaldo Luiz Caraciolo Ferreira ${ }^{4} \quad$ Lucineide de Jesus Teixeira $^{5}$
}

\begin{abstract}
RESUMO
A capacidade de regeneração natural de espécies nativas em situações de competição com árvores de plantios florestais pode ser considerado um fator de grande valor para a manutenção da diversidade. Diante disso, o presente estudo avaliou a regeneração natural de espécies nativas no sub-bosque de um povoamento de Eucalyptus saligna Smith., localizado na Reserva Biológica de Saltinho, PE. Para a análise da estimativa da regeneração natural das espécies arbóreas foram selecionados três talhões de Eucalyptus saligna Smith. Em cada um deles, foram implantadas parcelas de $1,0 \times 50 \mathrm{~m}$, sendo uma na extremidade do talhão e as outras distanciando $10 \mathrm{~m}$ de uma da outra, totalizando dez subunidades. As análises foram estabelecidas, com base no nível de inclusão das espécies arbóreas em regeneração natural com Circunferência do Caule a Altura de $30 \mathrm{~cm}$ do solo $-\left(\mathrm{CAB}_{0,30 \mathrm{~m}}\right) \leq 15 \mathrm{~cm}$, e as classes de alturas foram assim distribuídas: Classe 1 com indivíduos arbóreos com altura $(\mathrm{H}) 1,0 \leq \mathrm{H} \leq 2,0$, a Classe 2 com altura $(\mathrm{H}), 2<\mathrm{H} \leq 3$ e a Classe 3 com altura $(\mathrm{H})>3,0 \mathrm{~m}$. Para avaliar a diversidade florística a nível de espécie utilizou-se o índice de Shannon $\left(\mathrm{H}^{\prime}\right)$. No total, foram amostrados 302 indíviduos vivos, pertencentes a 23 famílias botânicas, 31 gêneros e 39 espécies. Analisando-se os resultados obtidos pode-se concluir que o eucalipto possibilita a regeneração de espécies nativas no sub-bosque, contudo, a composição florística depende também de características edáficas e da proximidade de fontes de propágulos.
\end{abstract}

Palavras-chave: regeneração natural; Floresta Ombrófila Densa; Eucalyptus saligna.

\begin{abstract}
The capacity of natural regeneration of native species in situations of competition with forest plantation trees can be considered an important factor in maintaining diversity. Therefore, the present study evaluated the natural regeneration of native species in the sub-forest of a settlement of Eucalyptus saligna Smith., located on the Biological Reservation of Saltinho - PE. To estimate the natural regeneration of the arboreal species, three stands of Eucalyptus saligna Smith were selected. In each one of them, 10 sub-units of $1.0 \mathrm{x}$ $50 \mathrm{~m}$ were implanted, one being on the edge of the stand and the others with a distance of $10 \mathrm{~m}$ one from

1. Bióloga, Mestre em Ciências Florestais, Departamento de Ciência Florestal, Universidade Federal Rural de Pernambuco, Rua J, 814, Paulo Camilo I, CEP 32530-260, Betim (MG). lidyalencar6@hotmail.com

2. Engenheiro Florestal, Dr., Professor do Departamento de Ciência Florestal, Universidade Federal Rural de Pernambuco, Av. Dom Manoel de Medeiros, s/n, Bairro Dois Irmãos, CEP 52171-900, Recife (PE). marangon@dcfl.ufrpe.br

3. Engenheira Florestal, Dra ${ }^{\mathrm{a}}$, Professora do Departamento de Ciência Florestal, Universidade Federal Rural de Pernambuco, Av. Dom Manoel de Medeiros, s/n, Bairro Dois Irmãos, CEP 52171-900, Recife (PE). licia@dcfl.ufrpe.br

4. Engenheiro Florestal, Dr., Professor do Departamento de Ciência Florestal, Universidade Federal Rural de Pernambuco, Av. Dom Manoel de Medeiros, s/n, Bairro Dois Irmãos, CEP 52171-900, Recife (PE). rinaldo@dcfl.ufrpe.br

5. Engenheira Agronoma, Mestre em Ciências Florestais, Superitêndencia de Meio Ambiente (SMA), Prefeitura Municipal de Salvador (PMS). Rua Leblon, 10E, Bairro São Cristóvão, CEP 41500-020, Salvador (BA). lucineide-texeira@ibest.com.br

Recebido para publicação em 18/03/2009 e aceito em 15/09/2010
\end{abstract}

Ci. Fl., v. 21, n. 2, abr.-jun., 2011 
another. The analyses were established based on the level of inclusion of the arboreal species in natural regeneration with circumference from stem to height of $30 \mathrm{~cm}$ from the soil $-\left(\mathrm{CAB}_{0.30 \mathrm{~m}}\right) \leq 15 \mathrm{~cm}$, and the classes of heights were distributed as follows: Class 1- arboreal individuals with heights $(\mathrm{H}) 1.0 \leq \mathrm{H} \leq$ 2.0; Class 2- with heights $(\mathrm{H}), 2<\mathrm{H} \leq 3$; and ; Class 3- with heights $(\mathrm{H})>3.0 \mathrm{~m}$. To evaluate the floristic diversity at the species level, the Shannon index was used $\left(\mathrm{H}^{\prime}\right)$. In total, 302 live individuals were sampled, belonging to 23 botanical families, 31 genera and 39 species. Based upon the results, it is possible to conclude that the Eucalyptus saligna tree does not preclude the natural regeneration of native species in the stand understory, however the floristic structure of the community is also influenced by edaphic factors and vicinity of seed sources.

Keywords: natural regeneration; Humid Forest; Eucalyptus saligna.

\section{INTRODUÇ̃̃O}

A regeneração natural, dependendo da conectividade da paisagem, pode proporcionar o estabelecimento de alta diversidade de espécies compondo uma gama de estratos vegetais, que são reconstituídos por interações planta-animal, conduzindo o sistema gradativo, para estágios sucessionais cada vez mais avançados (AIDE et al., 2000; UHL, et al., 2006).

Segundo Sartori et al. (2002), a capacidade de regeneração natural de espécies nativas em situações de competição com árvores de plantios florestais pode ser considerado um fator de grande valor para a manutenção da biodiversidade, visto que não só estaria mantendo o patrimônio genético vegetal, mas também estaria propiciando melhores condições de sobrevivência da fauna silvestre, assegurando melhores condições de abrigo e alimentação.

$\mathrm{O}$ processo de regeneração, que ocorre em uma floresta, dá-se por meio dos propágulos oriundos da dispersão, podendo as sementes ser autóctones ou alóctones, por intermédio do banco de sementes e plântulas encontradas no solo, e também pela propagação vegetativa ( RODRIGUES et al., 2004).

Estudos em plantações de Eucalyptus spp. e Pinus spp. caracterizam a formação de sub-bosque de espécies nativas, com base na regeneração natural. O estabelecimento dessas espécies pode se dar partindo de diásporos advindos de vegetação vizinha dos plantios, do banco de sementes ou da brotação de órgãos subterrâneos gemíferos, sobretudo em solos de Cerrado (AUBERT e OLIVEIRA FILHO, 1994). O fato de espécies de Eucalyptus não apresentarem nenhum indivíduo no estrato inferior do sub-bosque mostrou que as espécies nativas tiveram maior sucesso na regeneração natural. Eucalyptus grandis ao desenvolver o papel de pioneira favorece o desenvolvimento de uma comunidade jovem com característica de estádios avançados de sucessão como foi demonstrado por Silva Júnior et al. (1995).

Segundo CALEGARIO (1993), estudos de regeneração natural em plantios homogêneos podem fornecer subsídios importantes para 0 estabelecimento da vegetação com objetivo de recuperar áreas degradadas utilizando espécies exóticas, que por meio de práticas silviculturais seriam capazes de propiciar o desenvolvimento de povoamentos heterogêneos. A suposta esterilização do solo em plantios de eucalipto parece estar associada à imagem de povoamentos com pouca vegetação rasteira. Diferentes fatores, porém, podem estar relacionados a essa escassez de vegetação, sendo a baixa luminosidade, efeitos alelopáticos, espessura da manta orgânica e a concorrência por água e nutrientes frequentemente mencionados (REZENDE et al., 1994).

Apesar dos programas governamentais contemplarem plantios com espécies florestais nativas, a prática se limita à monocultura de Eucalyptus spp. em virtude do próprio interesse dos proprietários. Em razão da resistência dos produtores rurais ao plantio de espécies nativas para fins conservacionistas, fica evidente a importância dos estudos de regeneração natural como forma de viabilizar a recuperação da cobertura florestal. Plantios introduzidos por programas de fomento em fase de exploração têm apresentado significativo desenvolvimento de espécies florestais nativas no sub-bosque, sugerindo um processo sucessional favorável à recuperação da biodiversidade (REZENDE et al., 1994). Nesse contexto, as espécies de Eucalyptus spp. e Pinus spp. possuem um crescimento rápido e encontram-se em áreas plantadas no Brasil por possuírem um grande potencial madeireiro (IWAKIRI et al., 2000).

O objetivo deste trabalho foi avaliar a regeneração natural que ocorre em sub-bosque de Eucalyptus saligna Smith., visando a subsidiar recuperação de áreas degradadas de floresta 
ombrófila densa, na Reserva Biológica de Saltinho, PE.

\section{MATERIAL E MÉTODOS}

\section{Caracterização da Área}

\section{Localização}

O estudo foi desenvolvido na Reserva Biológica de Saltinho, PE, nos municípios de Tamandaré e Rio Formoso, que possui uma área de 475, 21 hectares. Os municípios de Tamandaré e Rio Formoso estão localizados entre as coordenadas $08^{\circ} 44^{\prime} 13^{\prime \prime}$ e $08^{\circ} 43^{\prime} 09^{\prime \prime}$ Latitude Sul e $35^{\circ} 10^{\prime} 11^{\prime \prime}$ e $35^{\circ} 11^{\prime} 02^{\prime \prime}$ Longitude Oeste (IBAMA, 2003).O clima predominante é do tipo tropical úmido (As') segundo a classificação de Köppen, no qual predomina as chuvas no outonoinverno, com precipitação pluviométrica de 1.500 $\mathrm{mm}$ onde está inserido os limites da Reserva, com temperatura média anual entre $22^{\circ} \mathrm{C}$ e $26^{\circ} \mathrm{C}$ (IBAMA, 2003).

\section{Solos}

Os solos predominantes na área geográfica do Município são classificados como latossolo vermelho-amarelo distrófico, com pequenas variações textuais como: argiloso, franco argiloso, argiloso arenoso e franco argilo arenoso (IBAMA, 2003).

\section{Vegetação}

No entorno da reserva predomina cultura extensiva de cana, sendo que a sua vegetação é constituída por formações secundárias florestais no domínio da Floresta Ombrófila Densa de Terras Baixas, em processo de regeneração natural e, por áreas de sistemas secundários, originários de plantio de espécies exóticas e nativas (IBAMA, 2003).

\section{Levantamento de Dados}

A vegetação do sub-bosque do povoamento de Eucalyptus saligna Smith. foi avaliada, utilizando-se o método de parcelas (FINOL, 1971; VOLPATO, 1994). Foram instaladas dez parcelas de 1 x 50 m, em três talhões Eucalyptus saligna Smith. Foram amostrados todos os indivíduos arbóreos com altura igual ou superior $1 \mathrm{~m}$. Desses indivíduos, foram tomadas medidas de diâmetro ao nível do solo e altura total, bem como coletado material botânico para identificação.
Os indivíduos mensurados receberam placas de PVC com numeração progressiva, as quais foram fixadas com linhas de nylon. Para a mensuração dos indivíduos que apresentaram altura até 2,0 metros foi utilizada uma trena de bolso, e para os indivíduos com altura superior, esta foi estimada com varas graduadas de $1,5 \mathrm{~m}$.

As análises foram estabelecidas, com base no nível de inclusão das espécies arbóreas em regeneração natural com Circunferência do Caule a Altura de $30 \mathrm{~cm}$ do solo $-\left(\mathrm{CAB}_{0,30 \mathrm{~m}}\right) \leq 15 \mathrm{~cm}$, e as classes de alturas foram assim distribuídas: Classe 1 com indivíduos arbóreos com altura $(\mathrm{H}) 1,0 \leq \mathrm{H} \leq$ 2,0, a Classe 2 com altura $(\mathrm{H}), 2<\mathrm{H} \leq 3$ e a Classe 3 com altura $(\mathrm{H})>3,0 \mathrm{~m}$, conforme (MARANGON, 1999).

Estabeleceu-se a altura mínima de 1,0 $\mathrm{m}$ para o estudo da regeneração natural avançada das espécies arbóreas, pois nessa, altura as espécies apresentam uma melhor definição de sua caracterização morfológica, permitindo assim uma identificação mais confiável.

Para avaliar a diversidade florística em nível de espécie, utilizou-se o índice de Shannon $\left(\mathrm{H}^{\prime}\right)$ conforme Felfili e Rezende (2003).

As espécies foram identificadas no campo, quando possível, e o material botânico foi coletado para posterior identificação por comparação com exsicatas do Herbário Sérgio Tavares (HST) do Departamento de Ciência Florestal da Universidade Federal Rural de Pernambuco - DCFL/UFRPE e Herbário Dardáno de Andrade Lima - IPA, da Empresa Pernambucana de Pesquisa Agropecuária. Para nomenclatura das espécies, seguiu-se o sistema de classificação de Cronquist (1988).

Foram avaliados os parâmetros fitossociológicos da regeneração em sub-bosques de Eucalyptus saligna Smith. Para cada espécie, foram estimados os parâmetros absolutos e relativos de frequência e densidade em cada classe de altura préestabelecida. Com base nesses parâmetros, estimouse a regeneração natural por classe de altura por meio das expressões que se seguem Silva et al. (2007).

$$
\mathrm{RNC}_{i j}=\frac{\mathrm{DR}_{i j}+\mathrm{Fr}_{i j}}{2}
$$

Em que:

$\mathrm{RNC}_{\mathrm{ij}}=$ estimativa da regeneração natural da i-ésima espécie na j-ésima classe de altura de planta, em percentagem; 
$\mathrm{DR}_{\mathrm{ij}}=$ densidade relativa para a i-ésima espécie na j-ésima classe de altura de regeneração natural;

$\mathrm{FR}_{\mathrm{ij}}=$ frequência relativa de i-ésima espécie, em percentagem, na j-ésima classe de regeneração natural.

Depois de calculadas a densidade e frequência (relativa e absoluta) de cada classe de altura, para cada espécie, estimou-se a regeneração natural, e foi utilizado o software Mata Nativa (CIENTEC, 2001), tanto para a regeneração natural por classe de altura como também para a regeneração natural total

Calculado o índice de regeneração por classe de altura para cada espécie, foi realizado o cálculo da estimativa da regeneração da comunidade amostrada por espécie, utilizandose da soma dos índices de regeneração natural por classe de altura conforme Silva et al.(2007).

$$
\mathrm{RNT}_{i}=\sum\left(\mathrm{RNC}_{i j}\right) / 3
$$

Em que:

$\mathrm{RNT}_{\mathrm{i}}=$ estimativa da regeneração natural total da população amostrada da i-ésima espécie;

$\mathrm{RNC}_{\mathrm{ij}}=$ estimativa da regeneração natural da i-ésima espécie na j-ésima classe de altura de planta.
Para análise da estrutura horizontal, utilizaram-se os seguintes parâmetros fitossociológicos: densidade absoluta e relativa, frequência absoluta e relativa, dominância absoluta e relativa e valor de importância, segundo a metodologia empregada por Felfili e Rezende (2003).

É importante salientar que, para se proceder à análise desse parâmetro, deve-se não só considerar os valores obtidos pelo somatório da densidade, frequência e dominância relativa da espécie, mas analisar esse valor junto a cada parâmetro que compõe sua fórmula isoladamente, caso contrário, pode-se estar cometendo um erro e/ou mascarando informações verídicas a respeito do grau de importância de cada espécie, comprometendo a sociologia, estrutura e ecologia da comunidade vegetal em estudo (FELFILI e REZENDE, 2003).

\section{RESULTADOS E DISCUSSÃO}

No levantamento da regeneração natural das espécies arbóreas nas parcelas implantadas nos talhões de Eucalyptus saligna Smith., foram amostrados 302 indíviduos vivos, pertencentes a 23 famílias botânicas, 31 gêneros e 39 espécies (Tabela 1). Dessas, 32 foram identificadas em nível de espécie, seis em nível de gênero e uma está como indeterminada.

TABELA 1: Espécies arbóreas, em ordem alfabética de família, encontradas nas parcelas de Eucalyptus saligna Smith., na Reserva Biológica de Saltinho.

TABLE 1: Arboreal species, in alphabetical order of family, found in the portions of Eucalyptus saligna Smith., on the Biological Reservation of Saltinho.

\begin{tabular}{ll}
\hline Família & Espécies \\
\hline Anacardiaceae & Tapirira guianensis Aubl. \\
& Thyrsodium spruceanum Benth. \\
Annonaceae & Guatteria oligocarpa Mart. \\
Apocynaceae & Himathantus phagedaenicus (Mart.) Woods. \\
Araliaceae & Schefflera morototoni (Aubl.) Maguine Steyerm \& Frondin \\
Burseraceae & Protium giganteum Engl \\
& Protium heptaphyllum (Aubl.) Marchand. \\
Caesalpiniaceae & Tachigali densiflora (Benth.) L.G. Silva \& H.C. Lima \\
Chrysobalanaceae & Hirtella racemosa Lam. \\
Clusiaceae & Licania tomentosa (Benth) \\
& Clusia nemorosa G. F. W. Meyer \\
Elaeocarpaceae & Rheedia gardneriana Planch. \& Triana. \\
\hline
\end{tabular}


TABELA 1: Cotinuação...

TABLE 1: Continued...

\begin{tabular}{|c|c|}
\hline Família & Espécies \\
\hline Erythroxylaceae & $\begin{array}{l}\text { Erythroxylum citrifolium A. St.-Hil. } \\
\text { Erythroxylum mucronatum Benth }\end{array}$ \\
\hline Euphorbiaceae & $\begin{array}{l}\text { Mabea sp. } \\
\text { Maprounea guianensis Aubl. } \\
\text { Margaritaria nobilis L. } \\
\text { Pogonophora schomburgkiana Miers ex Benth. } \\
\text { Richeria cf. grandis Vahl. }\end{array}$ \\
\hline Fabaceae & Lonchocarpus sericeus (Poir) Kunth ex DC. \\
\hline Lacistemataceae & $\begin{array}{l}\text { Lacistema } \text { cf. pubescens Mart. } \\
\text { Lacistema } \mathrm{sp} .\end{array}$ \\
\hline Lecythidaceae & Eschweilera ovata (Cambess.) Miers \\
\hline Melastomataceae & $\begin{array}{l}\text { Miconia ciliata }(\text { Rich.) DC. } \\
\text { Miconia prasina }(\text { Sw.) DC. } \\
\text { Miconia tomentosa (L.C.Rich.) Don. Ex DC. } \\
\text { Miconia sp. }\end{array}$ \\
\hline Meliaceae & Guarea kunthiana A. Juss. \\
\hline Mimosaceae & Inga laurina (Sw.) Willd. \\
\hline Moraceae & Brosimum conduru Standl. \\
\hline Myristicaceae & Virola gardneri (A.DC.) Warb. \\
\hline Myrtaceae & $\begin{array}{l}\text { Campomanesia dichotoma (Berg.) Mattos } \\
\text { Myrcia rhabdoides O. Berg. Vell. Aff. } \\
\text { Myrcia rostrata DC. } \\
\text { Myrcia } \mathrm{sp.}\end{array}$ \\
\hline $\begin{array}{l}\text { Simaroubaceae } \\
\text { Verbenaceae } \\
\text { Indeterminada A }\end{array}$ & $\begin{array}{l}\text { Simarouba amara Aubl. } \\
\text { Aegiphylla sp. } \\
\text { Indeterminada } 1\end{array}$ \\
\hline
\end{tabular}

As famílias que apresentaram maior destaque em número de espécies, ordenadas em ordem decrescente foram: Euphorbiaceae com cinco espécies, Melastomataceae e Myrtaceae com quatro espécies, Anacardiaceae, Burseraceae, Chrysobalanaceae, Clusiaceae, Erythroxylaceae e Lacistemataceae com duas espécies cada, juntas elas representaram $64,10 \%$ da regeneração natural avançada.

Nessa área, a família Melastomataceae está representada por quatro $\mathrm{sp}$., das quais pertencem ao gênero Miconia, indicando que essa maior riqueza pode ser decorrente do grau de perturbação que favorece o predomínio desse gênero conforme comentado por Tabarelli et al. (1997).

Sartori et al. (2002), analisando a regeneração natural em dois sítios, em sub-bosque de Eucalyptus saligna Smith., na estação experimental de Itatinga, São Paulo, observou as famílias com maior riqueza foram Myrtaceae (18 sp.), Solanaceae (9 sp.), Lauraceae (8 sp.), Euphorbiaceae (7 sp.), Fabaceae (6 sp.), Mimosaceae (5 sp.), Melatomataceae e Caesalpiniaceae ( 4 sp.).

Diversos outros autores trabalharam com estudos da regeneração natural em sub-bosque de Eucalyptus spp. em diferentes regiões do País, envolvendo áreas de domínios de Mata Altântica e Cerrado, que resultaram em uma forte indicação que as espécies regenerantes eram provenientes de formações florestais vizinhas (TABARELLI e MANTOVANII, 1999; SARTORI et al., 2002 e NERI et al., 2005).

No presente estudo, não foi diferente, a presença de Eucalyptus saligna Smith. não impossibilitou o crescimento de espécies nativas em seu sub-bosque.

Com relação a estudos já realizados no subbosque de povoamentos de Eucalyptus sp. Sartori et al. (2002), encontraram 107 espécies regenerando 
no sub-bosque em uma Estação Experimental de Ciências Florestais em Itatinga, São Paulo, se comparada com o presente estudo, considerase alto o valor de espécies encontradas. Valores bem inferiores foram registrados por Neri et al., (2005), encontraram 47 espécies regenerando no sub-bosque de Eucalyptus sp. em área de Cerrado na Floresta Nacional de Paraopeba, MG. Tubini (2006), encontrou 56 espécies regenerando no subbosque de Eucalyptus saligna emSão Bernardo do Campo, SP, após abandono de 13 anos.

Mochiutti et al. (2008), estudando a fitossociologia da regeneração natural em área de Acacia mearnsii De Wild., encontraram 49 espécies regenerando no sub-bosque no domínio da Floresta Estacional Semidecidual, no Rio Grande do Sul, após abandono de 16 anos.

No presente estudo, foram observadas 39 espécies regenerando no sub-bosque de Eucalyptus saligna, sem manejo há 22 anos.

Dentre as espécies que foram levantadas no estudo, as dez espécies mais presentes nos talhões de Eucalyptus saligna Smith. foram: Erythroxylum mucronatum, Miconia ciliata, Protium heptaphyllum, Licania tomentosa, Miconia prasina, Brosimum conduru, Eschweilera ovata, Rheedia gardneriana, Erythroxylum citrifolium e Lacistema cf. pubescens (Figura 1), que juntas, representam $79,47 \%$ dos indivíduos na área, e são as mais homogeneamente distribuídas, pois foram amostradas na maioria das parcelas.

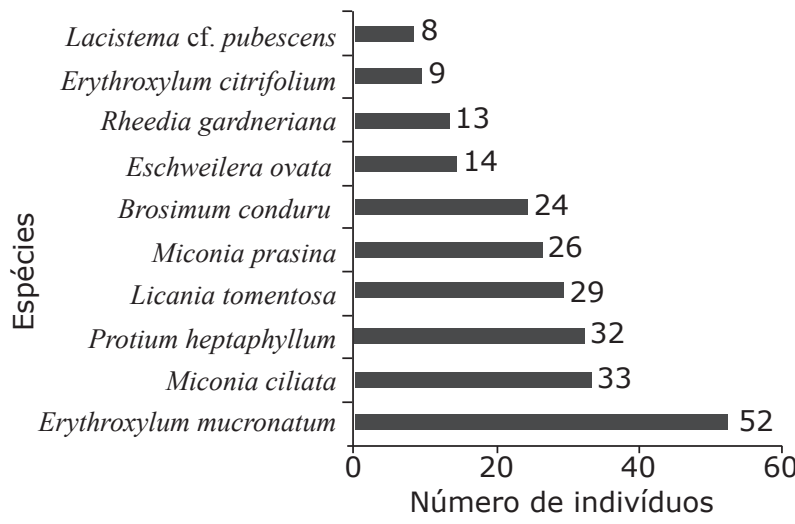

FIGURA 1: Espécies arbóreas e número de indivíduos amostrados nas parcelas de Eucalyptus saligna Smith. na Reserva Biologica de Saltinho, PE.

FIGURE 1: Arboreal species and number of individuals sampled in the portions of Eucalyptus saligna Smith. on the Biological Reservation of Saltinho, PE.
As dez espécies que apresentaram melhores desempenhos em termos de valor de importância (VI), para os talhões de E. saligna Smith. foram: Erytroxylum mucronatum, Miconia ciliata, Protium heptaphyllum, Miconia prasina, Eschweilera ovata, Brosimum conduru, Licania tomentosa, Rheedia gardneriana, Erythroxylum citrifolium e Miconia tomentosa (Figura 2).

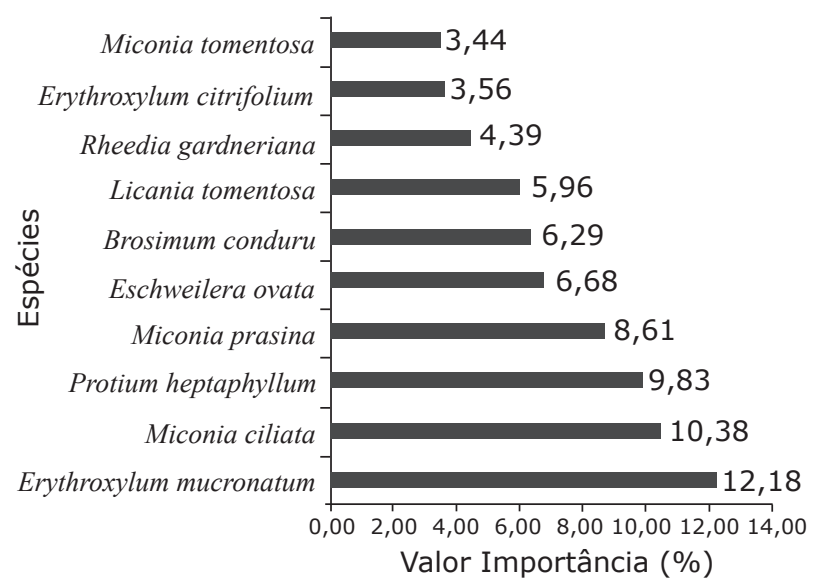

FIGURA 2: Valores percentuais de Importância (VI) das dez espécies arbóreas em processo de regeneração natural, ordenadas de forma decrescentes em Valor de Importância, nos talhões de Eucalyptus saligna Smith., na Reserva Biológica de Saltinho, PE.

FIGURE 2: Percentile Values of Importance (VI) of the ten arboreal species in process of natural regeneration, in decreasing order importance, in stands of Eucalyptus saligna Smith., on the Biological Reservation of Saltinho, PE.

As estimativas da regeneração natural total dentro das classes de altura (RNT) e a regeneração natural por classe de altura (RNC1, RNC2 e RNC3) com seus respectivos parâmetros fitossociológicos (densidades e freqüências relativas), estão representados na Tabela 2.

As espécies que apresentaram maiores índices de Regeneração Natural Total dentro das classes de altura (RNT), ordenados decrescentemente, foram: Miconia ciliata (Rich) DC. (11,81\%), Erythroxylum mucronatum Benth. (11, 48\%), Protium heptaphyllum (Aubl.) Marchand. (10,14\%), Miconia prasina (Sw.) DC. (8,02\%), Brosimum conduru Standl. (7,40\%),

Ci. Fl., v. 21, n. 2, abr.-jun., 2011 
TABELA 2: Estimativa da Regeneração Natural Total dentro das classes de altura (RNT) por espécie, bem como a estimativa da regeneração natural por classe de altura nas subunidades amostrais das parcelas de Eucalyptus saligna Smith., na Reserva Biológica de Saltinho, PE.

TABLE 2: Estimate of the Total Natural Regeneration within the height classes (RNT) for species, as well as the estimate of natural regeneration for height class in the sub-units sampled in stands of Eucalyptus saligna Smith., on the Biological Reservation of Saltinho, PE.

\begin{tabular}{|c|c|c|c|c|c|c|c|c|c|c|}
\hline Nome Científico & $\begin{array}{l}\mathrm{DR}_{1} \\
(\%)\end{array}$ & $\begin{array}{l}\mathrm{FR}_{1} \\
(\%)\end{array}$ & $\begin{array}{r}\mathrm{RNC}_{1} \\
(\%) \\
\end{array}$ & $\begin{array}{l}\mathrm{DR}_{2} \\
(\%)\end{array}$ & $\begin{array}{l}\mathrm{FR}_{2} \\
(\%)\end{array}$ & $\begin{array}{r}\mathrm{RNC}_{2} \\
(\%) \\
\end{array}$ & $\begin{array}{l}\mathrm{FR}_{3} \\
(\%)\end{array}$ & $\begin{array}{c}\mathrm{DR}_{3} \\
(\%)\end{array}$ & $\begin{array}{r}\mathrm{RNC}_{3} \\
(\%) \\
\end{array}$ & $\begin{array}{r}\text { RNT } \\
(\%) \\
\end{array}$ \\
\hline Miconia ciliata & 9,63 & 9,88 & 9,75 & 10,71 & 13,51 & 12,11 & 11,90 & 15,25 & 13,58 & 11,81 \\
\hline Erythroxylum mисronatum & 21,39 & 11,11 & 16,25 & 16,07 & 8,11 & 12,09 & 7,14 & 5,08 & 6,11 & 11,48 \\
\hline Protium heptaphyllum & 9,09 & 8,64 & 8,87 & 7,14 & 5,41 & 6,27 & 11,90 & 18,64 & 15,27 & 10,14 \\
\hline Miconia prasina & 9,09 & 6,17 & 7,63 & 5,36 & 5,41 & 5,38 & 11,90 & 10,17 & 11,04 & 8,02 \\
\hline Brosimum conduru & 8,56 & 6,17 & 7,36 & 10,71 & 10,81 & 10,76 & 4,76 & 3,39 & 4,08 & 7,40 \\
\hline Eschweilera ovata & 2,14 & 3,70 & 2,92 & 7,14 & 8,11 & 7,63 & 9,52 & 10,17 & 9,85 & 6,80 \\
\hline Licania tomentosa & 12,83 & 3,70 & 8,27 & 7,14 & 2,70 & 4,92 & 2,38 & 1,69 & 2,04 & 5,08 \\
\hline Rheedia gardneriana & 4,28 & 6,17 & 5,23 & 5,36 & 8,11 & 6,73 & 2,38 & 3,39 & 2,89 & 4,95 \\
\hline Lacistema cf. pubescens & 2,14 & 2,47 & 2,30 & 5,36 & 5,41 & 5,38 & 2,38 & 1,69 & 2,04 & 3,24 \\
\hline Miconia tomentosa & 2,14 & 3,70 & 2,92 & 1,79 & 2,70 & 2,24 & 4,76 & 3,39 & 4,08 & 3,08 \\
\hline Clusia nemorosa & 0,53 & 1,23 & 0,88 & 1,79 & 2,70 & 2,24 & 4,76 & 6,78 & 5,77 & 2,97 \\
\hline Guarea kunthiana & 0,00 & 0,00 & 0,00 & 7,14 & 5,41 & 6,27 & 2,38 & 1,69 & 2,04 & 2,77 \\
\hline Schefflera morototoni & 1,60 & 3,70 & 2,65 & 0,00 & 0,00 & 0,00 & 4,76 & 3,39 & 4,08 & 2,24 \\
\hline Erythroxylum citrifolium & 4,81 & 8,64 & 6,73 & 0,00 & 0,00 & 0,00 & 0,00 & 0,00 & 0,00 & 2,24 \\
\hline Maprounea guianensis & 0,53 & 1,23 & 0,88 & 1,79 & 2,70 & 2,24 & 2,38 & 3,39 & 2,89 & 2,00 \\
\hline Protium giganteum & 0,00 & 0,00 & 0,00 & 1,79 & 2,70 & 2,24 & 2,38 & 1,69 & 2,04 & 1,43 \\
\hline Thyrsodium spruceanum & 0,00 & 0,00 & 0,00 & 1,79 & 2,70 & 2,24 & 2,38 & 1,69 & 2,04 & 1,43 \\
\hline Lacistema $\mathrm{sp}$. & 0,00 & 0,00 & 0,00 & 0,00 & 0,00 & 0,00 & 4,76 & 3,39 & 4,08 & 1,36 \\
\hline Myrcia sp. & 1,60 & 1,23 & 1,42 & 1,79 & 2,70 & 2,24 & 0,00 & 0,00 & 0,00 & 1,22 \\
\hline Himathantus phagedaenicus & 0,53 & 1,23 & 0,88 & 1,79 & 2,70 & 2,24 & 0,00 & 0,00 & 0,00 & 1,04 \\
\hline Lonchocarpus sericeus & 0,53 & 1,23 & 0,88 & 1,79 & 2,70 & 2,24 & 0,00 & 0,00 & 0,00 & 1,04 \\
\hline Hirtella racemosa & 0,00 & 0,00 & 0,00 & 1,79 & 2,70 & 2,24 & 0,00 & 0,00 & 0,00 & 0,75 \\
\hline Sloanea sp. & 0,00 & 0,00 & 0,00 & 1,79 & 2,70 & 2,24 & 0,00 & 0,00 & 0,00 & 0,75 \\
\hline Aegiphylla sp. & 0,00 & 0,00 & 0,00 & 0,00 & 0,00 & 0,00 & 2,38 & 1,69 & 2,04 & 0,68 \\
\hline Margaritaria nobilis & 0,00 & 0,00 & 0,00 & 0,00 & 0,00 & 0,00 & 2,38 & 1,69 & 2,04 & 0,68 \\
\hline Simarouba amara & 0,00 & 0,00 & 0,00 & 0,00 & 0,00 & 0,00 & 2,38 & 1,69 & 2,04 & 0,68 \\
\hline Guatteria oligocarpa & 1,07 & 2,47 & 1,77 & 0,00 & 0,00 & 0,00 & 0,00 & 0,00 & 0,00 & 0,59 \\
\hline Tachigali densiflora & 1,07 & 2,47 & 1,77 & 0,00 & 0,00 & 0,00 & 0,00 & 0,00 & 0,00 & 0,59 \\
\hline Tapirira guianensis & 1,07 & 2,47 & 1,77 & 0,00 & 0,00 & 0,00 & 0,00 & 0,00 & 0,00 & 0,59 \\
\hline Campomanesia dichotoma & 0,53 & 1,23 & 0,88 & 0,00 & 0,00 & 0,00 & 0,00 & 0,00 & 0,00 & 0,29 \\
\hline Indeterminada 01 & 0,53 & 1,23 & 0,88 & 0,00 & 0,00 & 0,00 & 0,00 & 0,00 & 0,00 & 0,29 \\
\hline Inga laurina & 0,53 & 1,23 & 0,88 & 0,00 & 0,00 & 0,00 & 0,00 & 0,00 & 0,00 & 0,29 \\
\hline Mabea sp. & 0,53 & 1,23 & 0,88 & 0,00 & 0,00 & 0,00 & 0,00 & 0,00 & 0,00 & 0,29 \\
\hline Miconia sp. & 0,53 & 1,23 & 0,88 & 0,00 & 0,00 & 0,00 & 0,00 & 0,00 & 0,00 & 0,29 \\
\hline Myrcia rhabdoides & 0,53 & 1,23 & 0,88 & 0,00 & 0,00 & 0,00 & 0,00 & 0,00 & 0,00 & 0,29 \\
\hline Myrcia rostrata & 0,53 & 1,23 & 0,88 & 0,00 & 0,00 & 0,00 & 0,00 & 0,00 & 0,00 & 0,29 \\
\hline Pogonophora schomburgkiana & 0,53 & 1,23 & 0,88 & 0,00 & 0,00 & 0,00 & 0,00 & 0,00 & 0,00 & 0,29 \\
\hline Richeria cf. grandis & 0,53 & 1,23 & 0,88 & 0,00 & 0,00 & 0,00 & 0,00 & 0,00 & 0,00 & 0,29 \\
\hline Virola gardneri & 0,53 & 1,23 & 0,88 & 0,00 & 0,00 & 0,00 & 0,00 & 0,00 & 0,00 & 0,29 \\
\hline Total geral & 100 & 100 & 100 & 100 & 100 & 100 & 100 & 100 & 100 & 100 \\
\hline
\end{tabular}

Em que: DR = Densidade Relativa FR = Frequência Relativa, RNC 1 = Regeneração Natural da Classe 1 de Altura; RNC2 = Regeneração Natural da Classe 2 de Altura e RNC 3 = Regeneração Natural da Classe 3 de Altura. 
Alencar, A. L. et al.

Eschweilera ovata (Cambess.) Miers. (6,80\%), Licania tomentosa (Benth.) (5,08\%), Rheedia gardneriana Planch \& Triana (4,95\%), Lacistema cf. pubescens Mart. (3,24\%), e Miconia tomentosa (L. C. Rich.) Don. Ex DC. (3,08\%), Essas espécies juntas representam $72 \%$ da Regeneração Natural Total dentro das classes de altura para os talhões de Eucalyptus saligna Smith. e são as mais homogeneamente distruibuídas, pois foram amostradas em maior parte das parcelas.

Dentre as 39 espécies amostradas nas parcelas de Eucalyptus saligna Smith., as que apresentaram maiores índices em percentuais de Regeneração Natural na Classe 1 de altura (RNC1) em ordem decrescente foram: Erythroxylum mucronatum Benth. (5,42\%), Miconia ciliata (Rich) DC. (3,25\%) e Protium heptaphyllum (Aubl.) Marchand. (2,96\%), Miconia prasina (Sw.) DC. $(2,54 \%)$

Para a estimativa da Regeneração Natural na Classe 2 de altura (RNC2), as espécies que apresentaram maiores índices em percentuais ordenados de forma decrescente foram respectivamente: Miconia ciliata (Rich)

DC. $(4,04 \%), \quad$ Erythroxylum mucronatum Benth. (4,03\%), Brosimum conduru Staandl. (3,59\%) e Protium heptaphyllum (Aubl.) Marchand. (2,09\%).

As espécies que apresentaram maiores índices em percentuais de Regeneração Natural na Classe 3 de altura (RNC3) ordenadas decrescentemente foram: Protium heptaphyllum (Aubl.) Marchand. (5,09\%), Miconia ciliata (Rich) DC. $(4,53 \%)$, Miconia prasina (Sw.) DC. $(3,68 \%)$ e Eschweilera ovata (Cambess.) Miers. (3,28\%).

A maior densidade da regeneração natural encontrada neste trabalho pode ser atribuída à melhoria das condições ambientais do sub-bosque proporcionadas pela deposição de grande quantidade de serapilheira rica em nutrientes (SCHUMACHER et al., 2003).

$\mathrm{O}$ índice de diversidade de Shannon $\left(\mathrm{H}^{\prime}\right)$, normalmente, pode variar entre 1,3 e 3,5, podendo exceder 4,0 e alcançar 4,5 em ambientes florestais tropicais (FELFILI e REZENDE, 2003). O índice de diversidade encontrado para os talhões de Eucalyptus saligna Smith. foi de 2,86. Esse valor pode ser considerado alto, sobretudo, comparando-os com

TABELA 3: Índices de Shannon (H’) para espécies encontradas em sub-bosque de plantios comerciais de Eucalyptus sp.

TABLE 3: Shannon Index (H') for species found in sub-forest of commercial plantings of Eucalyptus sp.

\begin{tabular}{|c|c|c|c|c|c|c|c|}
\hline Local & Tipologia & Espécie & $\begin{array}{l}\text { Idade } \\
\text { (anos) }\end{array}$ & $\begin{array}{l}\text { Área } \\
\text { (ha) }\end{array}$ & Amostragem & $\mathrm{H}^{\prime}$ & Autor \\
\hline Belo Oriente & $\begin{array}{l}\text { Floresta Estacional } \\
\text { Semidecidual }\end{array}$ & $\begin{array}{l}\text { Eucalyptus } \\
\text { saligna }\end{array}$ & 6 & 0,04 & $\mathrm{CAP}>=5,0 \mathrm{~cm}$ & 2,20 & $\begin{array}{l}\text { Calegário e } \\
\text { Souza (1993) }\end{array}$ \\
\hline Itatinga & Cerrado & $\begin{array}{l}\text { Eucalyptus } \\
\text { saligna }\end{array}$ & - & 0,5 & Altura $>=1,5 \mathrm{~m}$ & 2,51 & $\begin{array}{l}\text { Sartori et al. } \\
(2002)\end{array}$ \\
\hline Itatinga & $\begin{array}{l}\text { Transição Floresta } \\
\text { Cerrado }\end{array}$ & $\begin{array}{l}\text { Eucalyptus } \\
\text { saligna }\end{array}$ & - & 0,5 & Altura $>=1,5 \mathrm{~m}$ & 3,75 & $\begin{array}{l}\text { Sartoni et al. } \\
(2002)\end{array}$ \\
\hline Paraopeba & Cerrado & Eucalyptus sp. & 30 & & & 2,49 & Neri et al. (2005) \\
\hline São Paulo & $\begin{array}{l}\text { Floresta Ombrófila } \\
\text { Densa }\end{array}$ & $\begin{array}{l}\text { Eucalyptus } \\
\text { saligna }\end{array}$ & 13 & & $\mathrm{PAP}>10 \mathrm{~cm}$ & 2,37 & Tubini (2006) \\
\hline $\begin{array}{l}\text { Tamandaré e } \\
\text { Rio Formoso }\end{array}$ & $\begin{array}{l}\text { Floresta Ombrófila } \\
\text { Densa de Terras } \\
\text { Baixas }\end{array}$ & $\begin{array}{l}\text { Eucalyptus } \\
\text { saligna }\end{array}$ & 22 & 0,5 & $\mathrm{CAP} \geq 15 \mathrm{~cm}$ & 2,86 & $\begin{array}{l}\text { Alencar et al. } \\
(2008)\end{array}$ \\
\hline
\end{tabular}

Ci. Fl., v. 21, n. 2, abr.-jun., 2011 
índices obtidos para diferentes tipologias florestais. Esse resultado mostra que o estabelecimento de espécies nativas em áreas de plantio com eucalipto e que a remoção do eucalipto certamente cria condições adequadas para o restabelecimento de comunidades secundárias nativas.

$\mathrm{Na}$ Tabela 3, são apresentados valores do índice de Shannon de outros estudos de regeneração natural de espécies nativas sob $o$ dossel de povoamentos de eucaliptos, os quais são de difícil comparação em razão das diferenças de área amostrada e limites de inclusão dos indivíduos, mas se pode verificar que os valores encontrados demonstram que as plantações de eucaliptos não impedem o desenvolvimento de espécies nativas no sub-bosque, mesmo considerando diferentes fases da sucessão.

Segundo Dias (2005), as florestas Tropicais muito pressionadas pelas ações antrópicas apresentam ecossistemas extremamente ameaçados incapazes muitas vezes de manterem a biodiversidade existente.

A variação nos valores do índice de diversidade apresentado deve-se sobretudo às diferenças nos estádios de sucessão somados às discrepâncias das metodologias de amostragem, níveis de inclusão, esforços de identificações taxonômicas além, das dissimilaridades florística das diferentes comunidades (Marangon, 1999).

Para Mochiutti et al. (2008), a diversidade da regeneração natural citada para diversas plantações florestais é de difícil comparação, por causa das diferenças metodológicas, como a área amostrada, limites para a inclusão de indivíduos, tamanho e idade do povoamento, fitogeografia da região, base logarítmica utilizada, etc. No entanto, a maioria dos valores de diversidade encontrados na literatura indica que essas plantações não impedem o desenvolvimento de espécies nativas em subbosque.

Apesar da dificuldade de comparações de índices de diversidade, o valor encontrado neste trabalho está na média aos citados para diversos povoamentos florestais, considerando as parcelas como um todo.

\section{CONCLUSÕES}

A regeneração natural em sub-bosque de Eucalyptus saligna Smith. é possível, desde que tais áreas tenham proximidade com diferentes fontes de propágulos.
Possívelmente, no atual estágio de estabelecimento das espécies arbóreas no subbosque de Eucalyptus saligna Smith., a remoção dos indivíduos irá acelerar o processo de regeneração proporcionando a continuidade da dinâmica florestal.

Eucalyptus saligna Smith. atuando como espécie pioneira, propiciou uma regeneração eficiente no aparecimento de espécies nativas.

O desenvolvimento de novos estudos e experimentações voltadas ao processo de regeneração natural em plantios comerciais pode contribuir para a adoção de melhores práticas de manejo, imprescindível para o estabelecimento de novas estratégias para a viabilização dos trabalhos de restauração.

\section{REFERÊNCIAS BIBLIOGRÁFICAS}

AIDE, T. M. et al. Forest regeneration in a chronosequence of tropical abondonded pastures implications for restoration ecology. Restoration Ecology, Malden, v. 8,n. 4,p. 328-338, Dec. 2000.

AUBERT, E. et al. Análise multivariada da estrututa fitossociológica do sub-bosque de plantios experimentais de Eucalyptus sp. e Pinus sp., em Lavras, MG, Revista Árvore, Viçosa, v. 18, n.3, p. 194- 214. 1994.

CALEGÁRIO, N. Parâmetros florísticos e fitossociológicos da regeneração natural de espécies arbóreas nativas no subbosque de povoamentos de Eucalyptus, no município de Belo Oriente, MG. 1993. 114 f. Dissertação (Mestrado em Ciência Florestal) - Universidade Federal de Viçosa, Viçosa, 1993.

CRONQUIST, A. The evolution and classification of flowering plants. New York: New York Botanical Garden, 1988. 555 p.

CIENTEC. Software Mata Nativa: manual do usuário. Viçosa: CIENTEC, 2001.131 p.

DIAS, A. C. Composição florística, fitossociologica e diversidade de espécies arbóreas e comparação de métodos de amostragem na Floresta Ombrófila Densa do Parque Estadual Carlos Botelho SP, Brasil. 2005. Tese (Doutorado em Recursos Florestais) - Escola Superior de Agricultura "Luiz de Queiroz", Piracicaba, São Paulo, 2005.

FELFILI, J. M.; REZENDE, R. P. Conceitos e métodos em fitossociologia. Brasília: Departamento de Engenharia Florestal da Universidade de Brasília - UnB, 2003. 68 p. 
FINOL, U. H. Nuevos parâmetros a considerarse en el analisis estrutural de las selvas virgenes tropicalis. Revista Forestal Venezolana, Mérida, v. 18, n. 12, p. 29-42. 1971.

IBAMA - Instituto Brasileiro do Meio Ambiente e dos Recursos Naturais Renováveis. Resumo executivo do plano de manejo da Reserva Biológica de Saltinho, fase 2. Brasília: IBAMA, 2003. 25 p.

IWAKIRI, S. et al. Avaliação da influência de diferentes composições de lâminas em compensados estruturais de Pinus elliottii e Eucalyptus saligna. Cerne, Lavras, v. 6, n. 2, p. 19-24. 2000.

MARANGON, L. C. Florística e fitossociologia de área de floresta estacional semidecidual visando dinâmica de espécies florestais arbóreas no município de Viçosa - MG. 1999. 139 f. Tese (Doutorado em Ecologia e Recursos Naturais) Universidade Federal de São Carlos, São Carlos, 1999.

MOUCHIUTTI, S. et al. Fitossociologia dos estratos arbóreo e de regeneração natural em um povoamento de acácia-negra (Acacia mearnsii De Wild.) na região da Floresta Estacional Semidecidual do Rio Grande do Sul. Ciência Florestal, Santa Maria, v. 18, n. 2, p. 207-222, abr./ jun. 2008.

NERI, A. V. et al. Regeneração de espécies nativas lenhosas sob plantio de Eucalyptus em área de cerrado na Floresta Nacional de Paraopeba, MG, Brasil. Acta Botanica Brasílica, São Paulo, v. 19, n. 2, p. 369-376, Oct. 2005.

REZENDE, M. L. et al. Regeneração natural de espécies florestais nativas em sub-bosque de Eucalyptus grandis e mata secundária no município de Viçosa, Zona da Mata - MG, Brasil. In: SIMPÓSIO SUL - AMERICANO, 1., SIMPÓSIO NACIONAL DE RECUPERAÇÃO DE ÁREAS DEGRADADAS, 2., 1994, Foz do Iguaçu. Anais... Foz do Iguaçu, PR. Curitiba: FUPEP, 1994. p. 409418.

RODRIGUES, R. R.; MARTINS, S. V.; BARROS, L. C. Tropical rain forest regeneration in na area degraded by mining, in Mato Grosso State, Brazil. Forest Ecology and Management, London, v. 190, p. 323-333, Oct. 2004.

SARTORI, M. S. et al. Regeneração da vegetação arbórea nativa no sub-bosque de um povoamento de Eucalyptus saligna Smith. localizado no Estado de São Paulo. Scientia Forestalis, Piracicaba, n. 62, p. 86-103,dez. 2002.

SILVA, W. C. et al. Estudo da Regeneração Natural de Espécies Arbóreas em Fragmento de Floresta Ombrófila Densa, Mata das Galinhas, no Município de Catende, Zona da Mata Sul de Pernambuco. Ciência Florestal, Santa Maria, v. 17, n. 4, p. 321331, out./dez. 2007.

SILVA JÚNIOR, M. C.; et al. Regeneration of na Atlantic Forest formation in the understorey of a Eucalyptus grandis plantation in south-eastern Brazil. Journal of Tropical Ecology, v. 11, p. 147152. 1995.

SCHUMACHER, M. V. et al. Retorno de nutrients via deposição de serapilheira em um povoamento de acácia-negra (Acacia mearnsii De Wild.) no Estado do Rio Grande do Sul. Revista Árvore, Viçosa, v. 27, n. 6, p.791-798, nov./dez. 2003.

TABARELLI, M.; MANTOVANI, W. A Regeneração de uma Floresta Tropical Montana após corte e queima (São Paulo-Brasil). Revista Brasileira de Biologia, Rio de Janeiro, v. 59, n. 2, p. 239-250, jun. 1999.

TABARELLI, M. et al. Colonização de clareiras naturais na floresta Atlântica no sudeste do Brasil.

Revista Brasileira de Biologia, Rio de Janeiro, v. 20, p. 57-66. 1997.

TUBINI, R. Comparação entre regeneração de espécies nativas em plantios abandonados de Eucalyptus saligna Smith. e em Fragmento de Floresta Ombrófila Densa em São Bernardo do Campo/SP. 2006. 94 f. Dissertação (Mestrado em Ecologia de Agroecossistemas)-Escola Superior de Agricultura "Luiz de Queiroz", Piracicaba, São Paulo, 2006.

UHL, C. et al. Abandoned pastures in Eastern Amazônia I. Patterns of plant sucession. The Journal of Ecology. Oxford, n, 76, p. 663-681. 2006.

VOLPATO, M. M. L. Regeneração natural em uma floresta secundária no domínio de Mata Atlântica: uma análise fitossociológica. 1994. 123 f. Dissertação (Mestrado em Ciência Florestal) - Universidade Federal de Viçosa, Viçosa, 1994. 